\title{
Centrality in Policy Network Drawings Extended Abstract
}

\author{
Ulrik Brandes ${ }^{1}$, Patrick Kenis ${ }^{2}$, and Dorothea Wagner ${ }^{1}$ \\ 1 University of Konstanz, Faculty of Mathematics and Computer Science \\ \{Ulrik.Brandes, Dorothea.Wagner\}@uni-konstanz.de \\ 2 Free University, Faculty of Social and Cultural Sciences, Amsterdam \\ p.kenis@scw.vu.nl
}

\begin{abstract}
We report on first results of a cooperation aiming at the usage of graph drawing techniques to convey domain-specific information contained in policy or, more general, social networks.

Policy network analysis is an approach to study policy making processes, structures and outcomes, thereby concentrating on the analysis of relations between policy actors. An important operational concept for the analysis of policy networks is centrality, i.e. the distinction of actors according to their importance in a relational structure. Matching structural with geometric centrality we incorporate the aggregated values of centrality measures into a layout model of the network.
\end{abstract}

\section{Introduction}

Given a graph from some area of application, utilization of general graph layout methods often yields drawings that are readable, but typically fail to communicate the important domain-specific information represented in the graph. Depending on the background, the same structure can clearly mean very different things, and different layouts can lead to different interpretations. To adhere to a fundamental principle of graphical excellence, that is to tell the truth about the data [16, p. 51], substance must therefore be considered already in the layout model.

We here report on a case study in which prominent domain-specific information contained in graphs from a sub-discipline of the social sciences is identified, and known layout models designed for readability of abstract graphs are adapted to account for substance. Complementary rendering rules are devised to contextually display further relevant information.

The case study presented originates from the field of public policy analysis. In the last decade, the policy network approach has become particularly prominent in the analysis of public policies. In contrast to other policy analysis approaches, the policy network approach has a much more realistic perspective of how policies develop, and considers policies principally as a result of a collaboration of a differentiated set of actors (public and private, local, regional and national, etc.). A policy network approach conceptualizes policy-making as the result of interactions between policy-actors, and assumes that the structure of 
these interactions explains policy outcomes [9]. A large number of structural characteristics is taken into account in the analysis and explanation of policy networks, but the most prominent one is certainly the notion of centrality. The reason being that public policy analysis is a sub-discipline of political science in which traditionally the principal question has been: "who has the power?". Centrality is considered a fairly good indicator for power in networks, and formalizations of the centrality concept are typically based on degree, shortest paths, or eigenvectors of graph related matrices [17].

Except for an isolated historical example [13], we know of no attempt to visualize centrality, or, for that matter, any other structural variable of a network, and the underlying network at the same time. This is despite the fact that empirical studies stress the effect of layout on the perception of structural characteristics [11]. Note that structural centrality has an immediate geometric pendant. Though layout methods used for social networks in general, like multidimensional scaling and spring embedder variants [10,1], occasionally do a fairly good job of placing central actors close to the center, they are by no means reliable in this respect, but often misleading. We here show how to exactly represent the aggregated values together with the underlying network data.

In Sect. 2, we provide some background on the area of application, and develop a layout model and rendering rules in Sect. 3. Suitability of the resulting drawing model was established in an exploratory data analysis from which examples are presented in Sect. 4 .

\section{Policy Networks and Centrality}

Centrality is considered a fundamental characteristic of policy networks since it gives an indication of the most important, the important and the unimportant actors in the network. The question is particularly relevant for mainly two reasons: first it tells us something about the social or political structure of policy making and secondly it helps understanding the outcomes that policy networks produce. The social or political structure of a network indicates which type of actor is involved in which way in the policy-making process. Who has access and control over resources and who has a brokerage position? From this perspective it makes considerable difference whether there is a most important actor in a network and, if this is the case, what type of actor it is. In terms of legitimacy, accountability, justice, etc. it makes a difference whether in the health policy field, for example, the most important actor is a state or a private actor and how important the actor is relative to the others. Moreover, there is evidence that the centrality structure of the network explains why a network was particularly successful in producing certain outcomes, or why policies have failed to come about.

A number of approaches have been devised to operationalize "importance", all of which are equally accepted because they address different dimensions of the intuitive notion (for an overview see $[6,7]$ ). We limit our exposition to three exemplary measures that are used widely: degree, closeness, and betweenness centrality. 
The degree centrality, $C_{D}(v)$, of a vertex $v$ is simply the degree of that vertex, the idea being that the degree to which actors are active in relating to other actors is relevant. The actors with a high degree centrality are where the action in the network is. In policy networks these actors are highly visible for the other actors and are recognized by the others as a major channel of relational information.

This very local measure can be extended in several ways to take into account the whole graph. One is by taking the closeness, i.e. the sum of the distances to all other vertices, as a basis. Closeness centrality, defined as the inverse closeness, $C_{C}(v)=\left(\sum_{u \in V} d_{G}(u, v)\right)^{-1}$, focuses on how close an actor is to all the other actors in the network. If we consider a policy network where a certain actor has information which is crucial to all other actors one would expect this actor to have a high closeness centrality for the network to function effectively.

Finally, betweenness centrality is defined as the sum of the ratios of shortest paths between other actors that an actor sits on, $C_{B}(v)=$ $\sum_{u \neq v \neq w}\left|P_{v}(u, w)\right| /|P(u, w)|$, where $P(u, w)$ and $P_{v}(u, w)$ are the sets of all shortest paths between vertices $u$ and $w$, and those shortest paths passing through $v$, respectively. It indicates which other actors have control over the interaction between two non-adjacent actors. An actor with high betweenness centrality is between many actors in terms of shortest paths. In policy networks these actors are considered important because they control the spread of information between actors or sets of actors and thus can influence decision-making processes.

A framework to obtain normalized and network level centrality measures from a given actor level measure is described in [6]. Any centrality measure $C$ is normalized to lie between zero and one by dividing its values by the maximum possible score in any graph with the same number of vertices. The above three measures thus yield normalized measures $C_{D}^{\prime}(v)=\frac{C_{D}(v)}{n-1}, C_{C}^{\prime}(v)=\frac{C_{C}(v)}{1 /(n-1)}$, and $C_{B}^{\prime}(v)=\frac{C_{B}(v)}{(n-1)(n-2) / 2}$. Network centralization on the other hand quantifies the range of variability of the individual actor indices. A low network centralization in a policy network is thus an indication that there is not a clear center of action. Centralization is formalized as the cumulated differences between actor centralities and the maximum score attained in the present network, normalized by the maximum possible such sum. For all of the above measures, the star is a maximally centralized graph, whereas cliques and circles are not centralized at all.

The network in Fig. 1 shows that these measures actually differ. Each identifies a different set of maximally central vertices, marked by corresponding labels.

\section{Centrality Drawings}

Drawings of policy networks are to aid the exploration and communication of substantive content. Since centrality is such an important concept in policy network analysis, we want to devise means to make it visible in a graphical presentation of the policy network, rather than listing scores in a table (as is common). 
Following [2], we think of graphical presentations as composed of graphical primitives, called graphical features (points, lines, areas, volumes), that represent data elements. These features have properties, called graphical variables (size, shape, color, etc.), that are either fixed according to a chosen form of representation, or varied according to the data. To produce an effective graph visualization, three main aspects have to be taken into account [3]:

- The graph's substance, i.e. the syntactic (intrinsic) and semantic (extrinsic) domain-specific information that is to be represented.

- The graphical design specifying which graphical features are to represent which data elements (representation), and how values shall be assigned to positional (layout) and retinal (rendering) graphical variables.

- The algorithm used to determine the layout, since many criteria of good graphical design are only approximately satisfiable and a particular algorithm may thus introduce particular artifacts.

Our representation of choice is the traditional sociogram [12]. In an attempt to make substance visible, our layout model maps structural to geometric centrality. We thus constrain each vertex to lie on a circle centered at the center of the diagram. The radius $r(v)$ of the circle for vertex $v$ is determined from the structural centrality $C(v)$ of $v$. After experimenting with several other mappings, we decided to use radii

$$
r(v)=1-\frac{C^{\prime}(v)-\min _{u \in V} C^{\prime}(u)}{\max _{u \in V} C^{\prime}(v)-\min _{u \in V} C^{\prime}(u)+c(G)}
$$

where $c(G)$ is an offset used to avoid overlap if there is more than one vertex of maximum centrality. Showing levels as thin circles allows to compare centrality scores exactly, so that an accompanying tabular presentation is no longer needed.

The remaining degrees of freedom in the layout model should be used to ensure readability of the diagram. Unfortunately, the barycentric technique used for ring diagrams of hierarchies [14] is not suited for our problem, because transitive edges (not present in a hierarchy) may result in edge overlap.

The circular layering is also reminiscent of layered drawings of directed graphs (as in [15]), and a small number of edge crossing is a natural requirement is both cases. However, the number of crossings even between two centrality levels in a radial layout is not uniquely determined by the cyclic orderings of vertices on these levels. This observation still holds, if positions in one layer are fixed. By necessity, radial differences between centrality levels are fixed. Note that this is different from the usual approach to layered drawings of directed graphs, where layer distances are computed only after determining the orderings of vertices on each level. Fixed level differences suggest to impose the additional constraint that edges between two levels may not pass through the inner circle (outward drawing). Crossing minimization is then easily reduced to two-layer crossing minimization with one fixed layer, which is $\mathcal{N} \mathcal{P}$-complete [5]. Moreover, bend edges (necessarily introduced by two-layer methods) tend to be rather confusing in radial layouts. We thus decided not to try adaptations of methods devised for layered layouts. 
To try out the effectiveness of different designs, we instead exploited the flexibility of energy-based placement approaches. Our prevailing objective function uses the pairwise spring potentials of [8], where preferred distances are determined from shortest paths in the underlying graph, where edge lengths are redefined according to level span and number of actors on similar levels. Additionally, we used vertex-edge repulsion and crossing counts similar to those in [4]. To achieve better overall organization, the layout procedure employs three stages working on different subgraphs, and the various penalties are dependent on the annealing temperature. Details appear in the full paper.
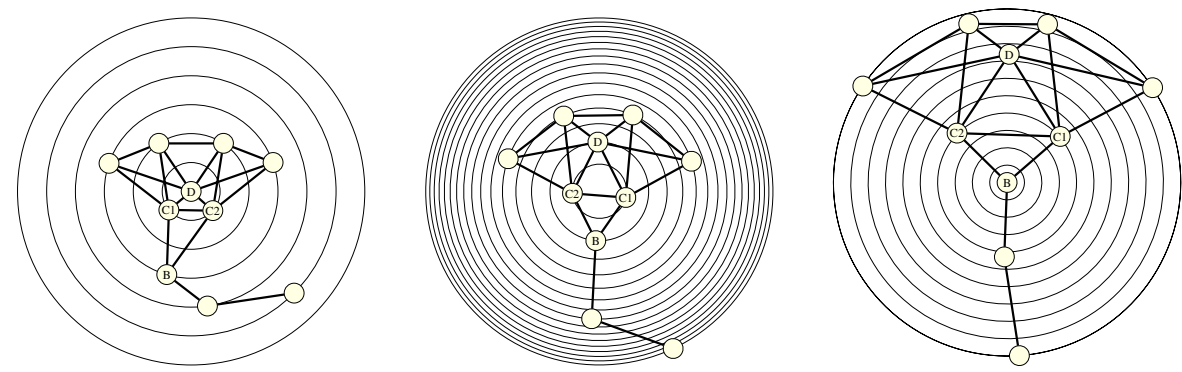

Fig. 1. Layouts showing degree, closeness, and betweenness centrality, respectively

While the layout is designed to organize the network in a readable way that exactly represents actor centralities, we can use the remaining graphical variables to convey further substance. Due to space limitations we briefly skim over them. In the examples below we have coded legal status and attitudes towards the issue by shape and color of vertices, respectively. Confirmed relations are drawn as thick black lines, whereas unconfirmed relations are drawn as grey lines with an arrow indicating who mentioned whom (see below). In the directed network of claimed relationships, the ratio of the in- and outdegree of each vertex measures the reciprocation of an actor's claims. We therefore vary the size of point features representing actors so that the ratio of height and width equals the ratio of inand outdegree, while the area is proportional to the combined degree.

\section{Analyzing Local Drug Policies}

In this final part the usefulness of the graph drawing techniques presented above will be demonstrated. The demonstration is based on data from a project studying the incidence of HIV-preventive measures for IV-drug users in 9 German municipalities. The research question underlying the project is: why differ these municipalities so much in the provision of HIV-preventive measures (such as methadone substitution and needle exchange) given the fact that the problem load (i.e. the number of IV drug users and the HIV epidemiological situation) are very similar? The study tests the hypothesis whether the difference in the 
provision in HIV-preventive measures can best be explained by the structure of the policy networks. Given the amount of controversy and complexity involved in the provision of such measures the hypotheses is that they are contingent on types of relations between the different policy actors. The policy networks studied here include all local organizations directly or indirectly involved in the provision of such measures.

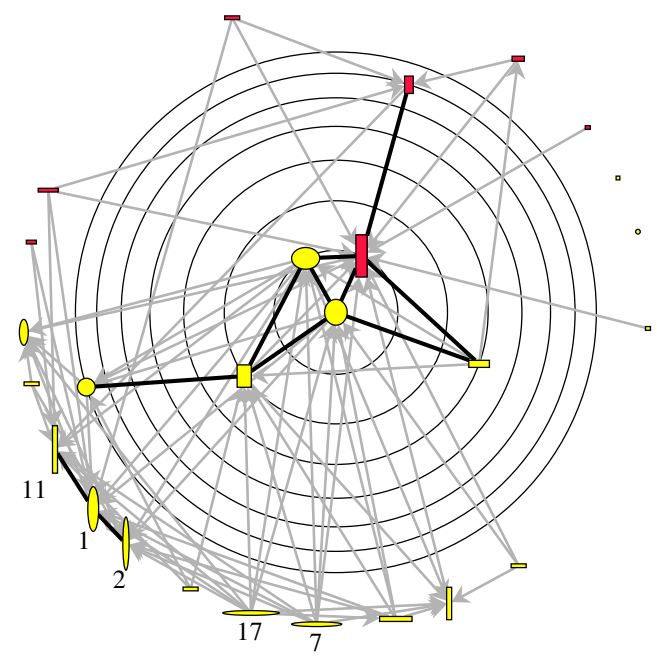

Fig. 2. Organizations involved in local drug policy making in Stuttgart

To sketch how a drawing can elucidate structural aspects of a network, we first take a look at the network of informal communication in the city of Stuttgart (Fig. 2). Informal communication is in principle an undirected relationship: if $A$ talks to $B$, you assume that $B$ also talks to $A$. What you see very often in empirical investigations, however, is that many undirected relationships are not reciprocated: although $A$ mentioned $B$ as a communication partner, $B$ did not mention $A$. Consequently, policy network analysts often make a principal decision and either decide to calculate centrality on the basis of only confirmed or of both confirmed and unconfirmed relationships. In contrast, an analysis of this phenomenon on the basis of the drawing presented tells a number of additional stories. While the unconfirmed network appears fairly integrated, the confirmed network is sparse. The drawing however reveals that there is a crucial separation due to actors 1,2 , and 11 , who are peripheral only because they seem reluctant to name others. Actors 7 and 17 (small non-profit organizations) are also interesting since they claim a huge number of relationships, none of which are reciprocated. Consequently, on the sole basis of these drawings a lot of additional facts are provided which help to come to an adequate evaluation of who actually the most important actor is. 
As a second example we demonstrate the use of imaging in the analysis of the relationship between network structure and network outcome (i.e. in the present case the provision of HIV-preventive measures). The three networks presented in Fig. 3 (Essen, Köln, and Ahlen) differ a lot in their outcome effectiveness (Essen being the most and Ahlen being the least effective) but also differ a lot in their structural characteristics with respect to different measures of centrality.

On the basis of the drawings a number of additional relationships become visible which can be further developed regarding the effectiveness of networks: the number of rather active actors (i.e. with high degree centrality); the degree of discrepancy between confirmed and unconfirmed links; the fact whether a mixture of different types of actors (public and private, and repressive and supportive) are found close the center of the network; the fact whether there is one clearly central actor in the network, a couple of central actors, or no clearly central actors in the network. It is this type of inductive observations, which are a direct result of the graph drawing techniques and which contribute substantially to the analysis of the questions "who has the power?" and "what are the consequences of the power structure?".

Acknowledgment. We thank Vanessa Kääb for providing several earlier implementations of different centrality measures and experimental layout models.

\section{References}

1. Vladimir Batagelj and Andrej Mrvar. PAJEK - Program for large network analysis. Connections, 21:47-57, 1998.

2. Jacques Bertin. Semiology of Graphics: Diagrams, Networks, Maps. University of Wisconsin Press, 1983.

3. Ulrik Brandes, Patrick Kenis, Jörg Raab, Volker Schneider, and Dorothea Wagner. Explorations into the visualization of policy networks. Journal of Theoretical Politics, 11(1):75-106, 1999.

4. Ron Davidson and David Harel. Drawing graphs nicely using simulated annealing. ACM Transactions on Graphics, 15(4):301-331, 1996.

5. Peter Eades and Nicholas C. Wormald. Edge crossings in drawings of bipartite graphs. Algorithmica, 11:379-403, 1994.

6. Linton C. Freeman. Centrality in social networks: Conceptual clarification. Social Networks, 1:215-239, 1979.

7. Noah E. Friedkin. Theoretical foundations for centrality measures. American Journal of Sociology, 96(6):1478-1504, May 1991.

8. Tomihisa Kamada and Satoru Kawai. An algorithm for drawing general undirected graphs. Information Processing Letters, 31:7-15, 1989.

9. Patrick Kenis and Volker Schneider. Policy networks and policy analysis: Scrutinizing a new analytical toolbox. In Bernd Marin and Renate Mayntz, editors, Policy Networks: Empirical Evidence and Theoretical Considerations, pages 25-59. Campus Verlag, 1991.

10. David Krackhardt, Jim Blythe, and Cathleen McGrath. KrackPlot 3.0: An improved network drawing program. Connections, 17(2):53-55, 1994.

11. Cathleen McGrath, Jim Blythe, and David Krackhardt. The effect of spatial arrangement on judgments and errors in interpreting graphs. Social Networks, 19(3):223-242, 1997. 


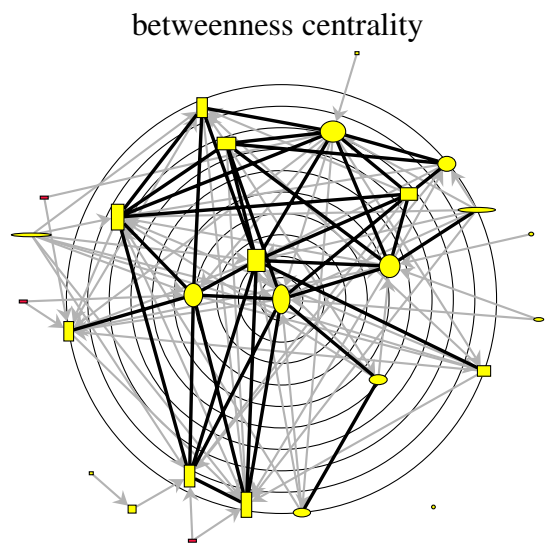

Essen
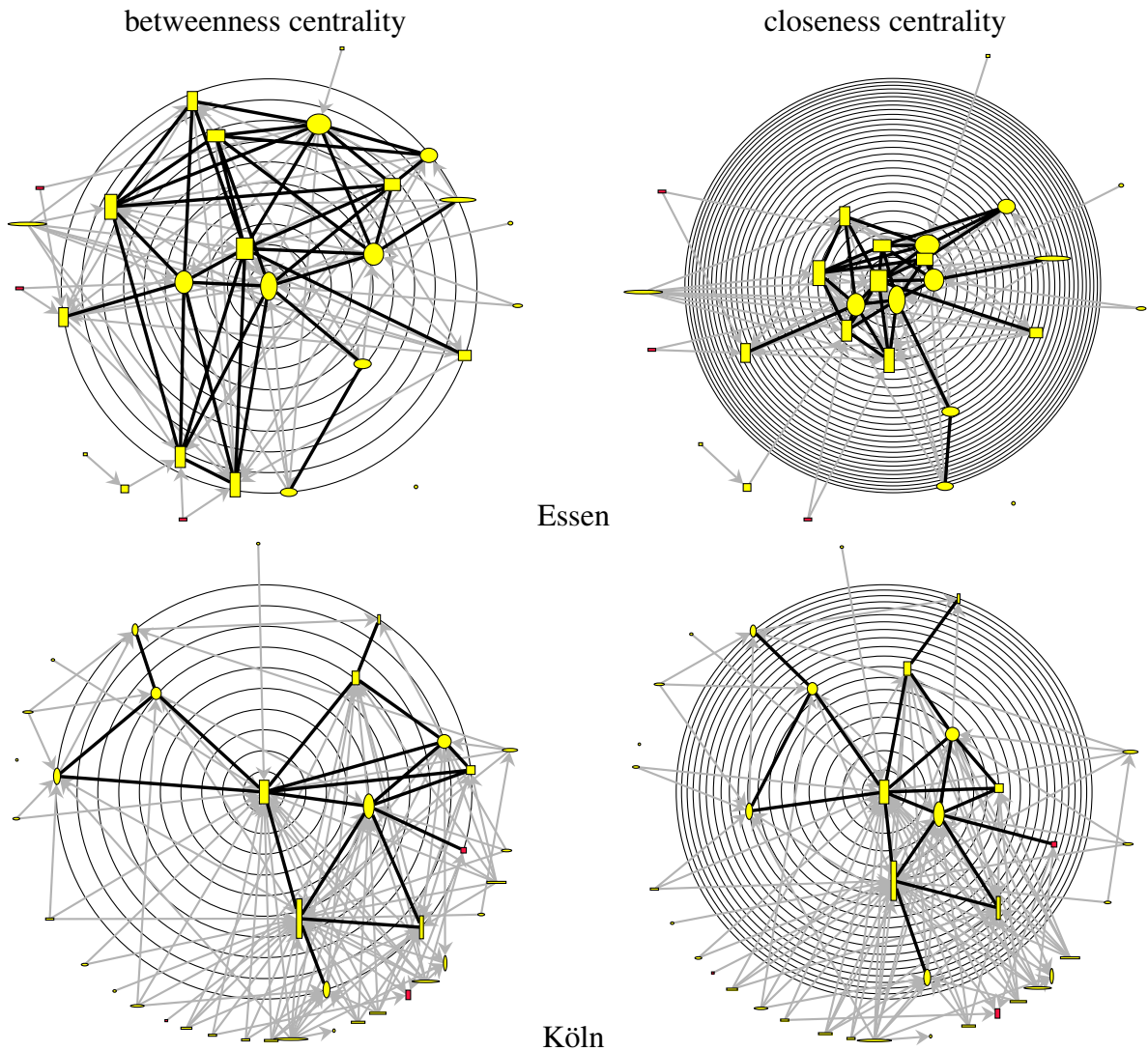

Köln
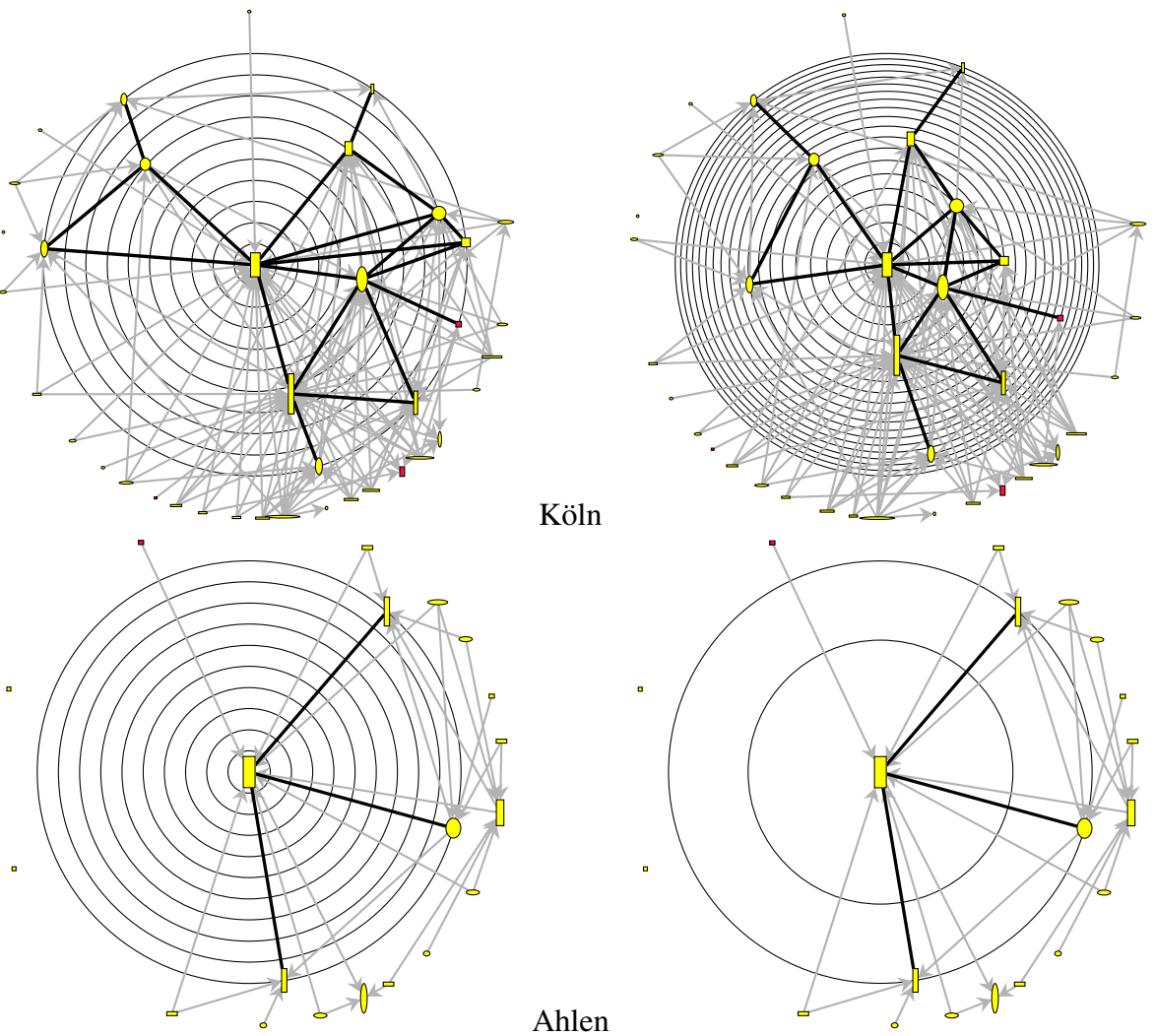

Fig. 3. Networks of informal communication. Centralities are according to confirmed networks, while vertex size codes normalized in- and outdegree centrality in unconfirmed networks and is comparable across drawings 
12. Jakob L. Moreno. Who Shall Survive: Foundations of Sociometry, Group Psychotherapy, and Sociodrama. Beacon House, 1953.

13. Mary L. Northway. A method for depicting social relationships obtained by sociometric testing. Sociometry, 3:144-150, 1940.

14. Marcello G. Reggiani and Franco E. Marchetti. A proposed method for representing hierarchies. IEEE Transactions on Systems, Man and Cybernetics, 18(1):2-8, 1988.

15. Kozo Sugiyama, Shojiro Tagawa, and Mitsuhiko Toda. Methods for visual understanding of hierarchical system structures. IEEE Transactions on Systems, Man and Cybernetics, 11(2):109-125, February 1981.

16. Edward R. Tufte. The Visual Display of Quantitative Information. Graphics Press, 1983.

17. Stanley Wasserman and Katherine Faust. Social Network Analysis: Methods and Applications. Cambridge University Press, 1994. 